\title{
Bounded-Error Identification of Linear Systems with Input and Output Backlash
}

\author{
V. Cerone ${ }^{1}$, D. Piga $^{2}$, D. Regruto ${ }^{1}$
}

${ }^{1}$ Dipartimento di Automatica e Informatica, Politecnico di Torino, Italy

2 Delft Center for Systems and Control, Delft University of Technology, The Netherlands

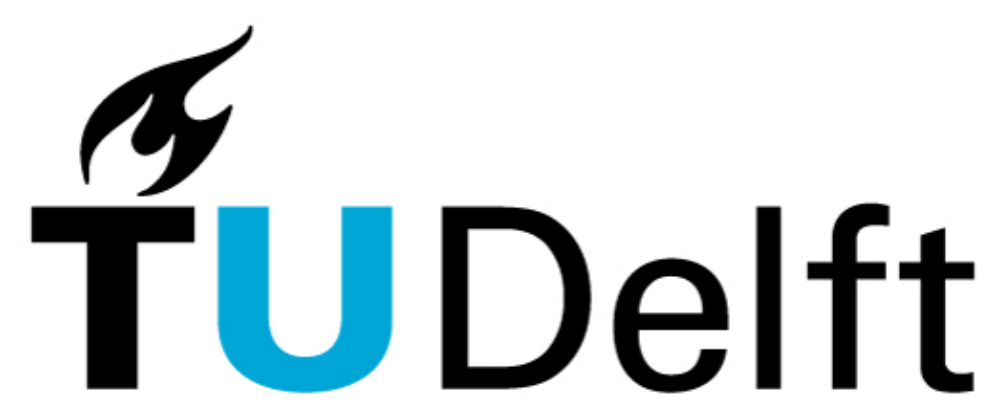

Technische Universiteit Delft

16th IFAC Symposium on System Identification

Session WeB01: "Block Oriented Nonlinear Identification 2"

Brussels, Belgium - July 11, 2012 


\section{System Description}

Linear dynamical system with input backlash

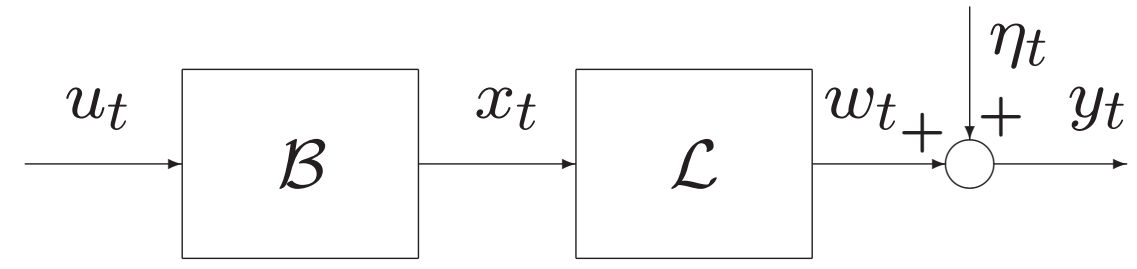

Linear dynamical system with output backlash

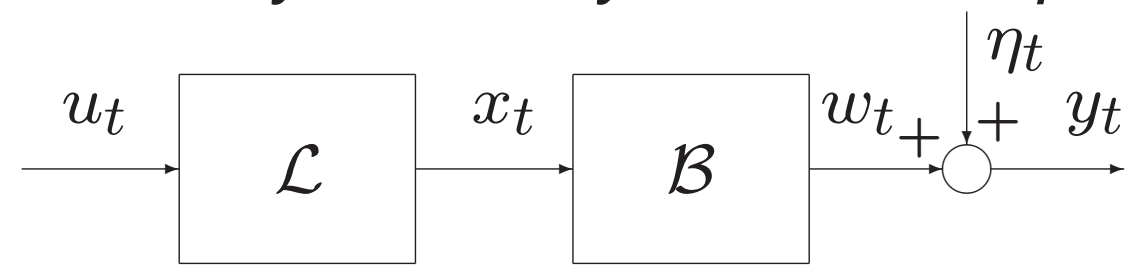

$\left|\eta_{t}\right| \leq \Delta \eta_{t} ; \quad \Delta \eta_{t}$ known (Set-Membership characterization) $u_{t}$ : known input signal

$y_{t}:$ noise-corrupted measurement of $w_{t}$

$x_{t}$ : not measurable inner signal

$\mathcal{B}$ : backlash nonlinearity

$\mathcal{L}$ : linear dynamic subsystem 


\section{System Description}

$$
\stackrel{u_{t}}{\longrightarrow \mathcal{L}} \stackrel{x_{t}}{\longrightarrow \mathcal{B}} \stackrel{w_{t}}{\rightarrow} \stackrel{\eta_{t}}{\longrightarrow} y_{t}
$$

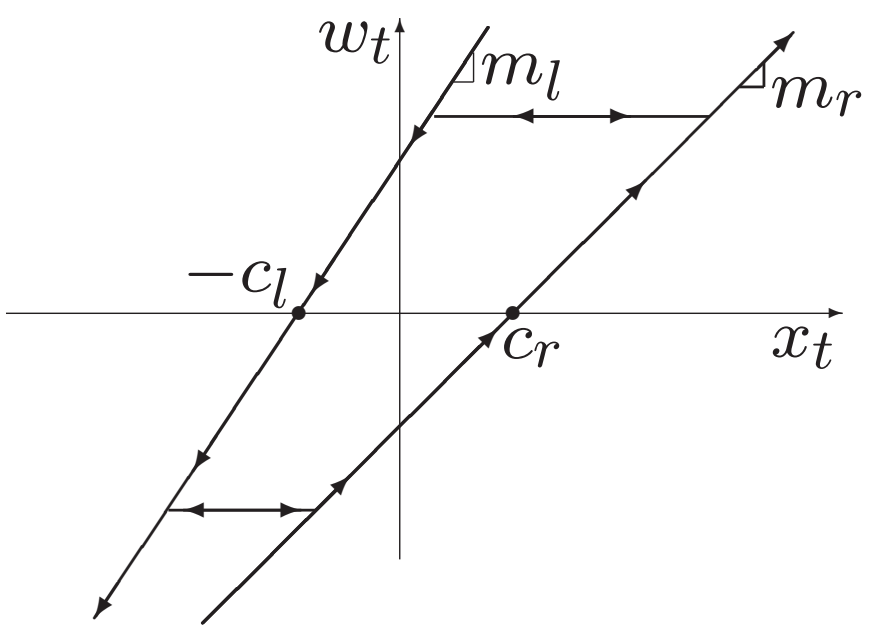

$$
w_{t}=\mathcal{B}\left(x_{t}\right)= \begin{cases}m_{l}\left(x_{t}+c_{l}\right) & \text { for } x_{t} \leq \frac{w_{t-1}}{m_{l}}-c_{l} \\ m_{r}\left(x_{t}-c_{r}\right) & \text { for } x_{t} \geq \frac{w_{t-1}}{m_{r}}+c_{r} \\ w_{t-1} & \text { for } \frac{w_{t-1}}{m_{l}}-c_{l}<x_{t}<\frac{w_{t-1}}{m_{r}}+c_{r}\end{cases}
$$

$$
\mathcal{L}: x_{t}=-\sum_{i=1}^{n a} a_{i} x_{t-i}+\sum_{j=0}^{n b} b_{j} u_{t-j}
$$




\section{Identification of linear systems with backlash}

- Aim of the work: compute bounds on the backlash parameters $\gamma^{\top}=\left[m_{l} c_{l} m_{r} m_{r}\right]$ and linear block parameters $\theta^{\top}=\left[\begin{array}{lllllll}a_{1} & \ldots & a_{n a} & b_{0} & b_{1} & \ldots & b_{n b}\end{array}\right]$.

- Parameter bound computation of linear systems with backlash is NP-hard in the size of the experimental data sequence

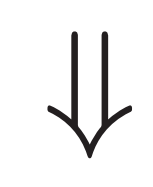

Computationally tractable relaxations are needed 


\section{Feasible parameter set (FPS)}

In bounded-error (or set-membership) context, all the system parameters $\gamma$ and $\theta$ consistent with the measurement data sequence, the assumed model structure and the error bounds are feasible solution to the identification problem (and are said to belong to the feasible parameter set $\left.\mathcal{D}_{\gamma \theta}\right)$.

\section{How to construct the Feasible Parameter Set?}




\section{Backlash nonlinearity}

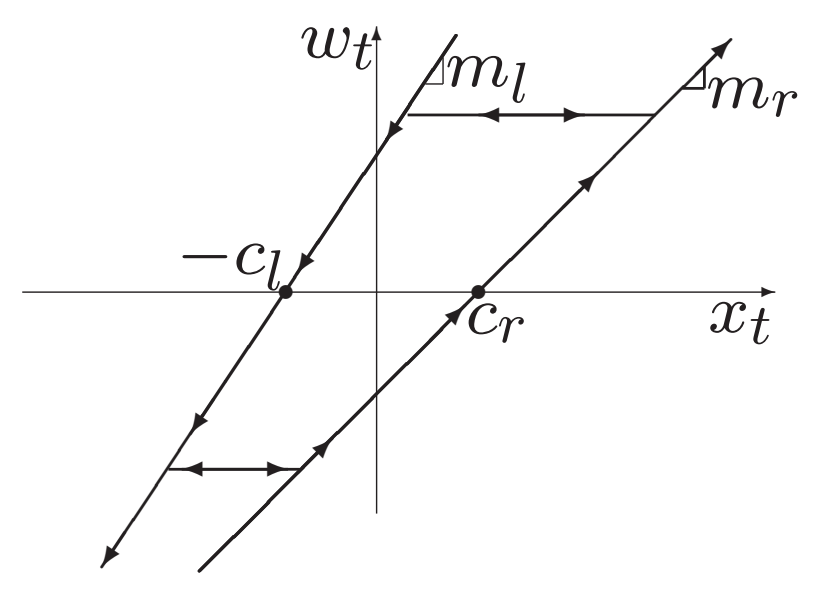

Can the backlash nonlinearity be inverted? 


\section{Backlash nonlinearity}

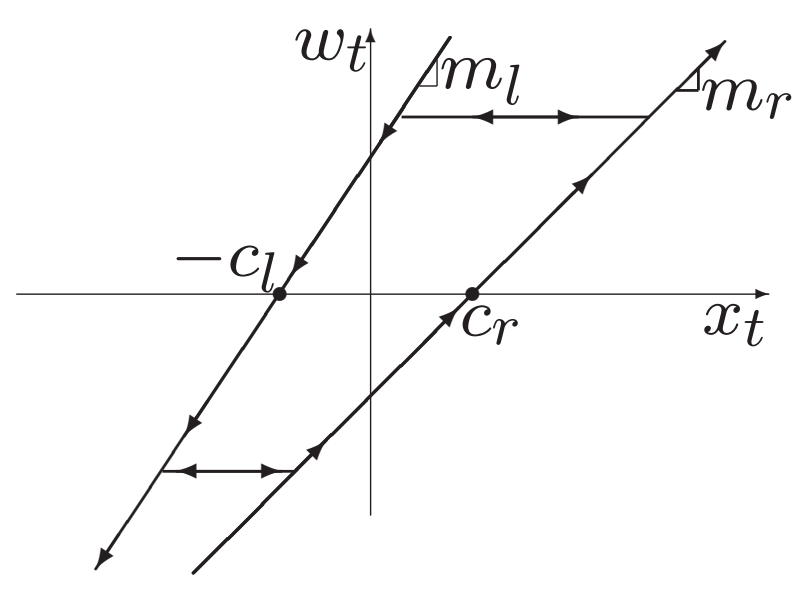

Definition 1: $\mathcal{Y}^{r}$ (right-invertible output sequence)

$$
\mathcal{Y}^{r}=\left\{y_{t} \in \mathbb{R}: y_{t}-y_{t-1}>\Delta \eta_{t}+\Delta \eta_{t-1}\right\}
$$

Definition 2: $\mathcal{Y}^{l}$ (left-invertible output sequence)

$$
\mathcal{Y}^{l}=\left\{y_{t} \in \mathbb{R}: y_{t}-y_{t-1}<-\Delta \eta_{t}-\Delta \eta_{t-1}\right\}
$$




\section{Backlash nonlinearity}

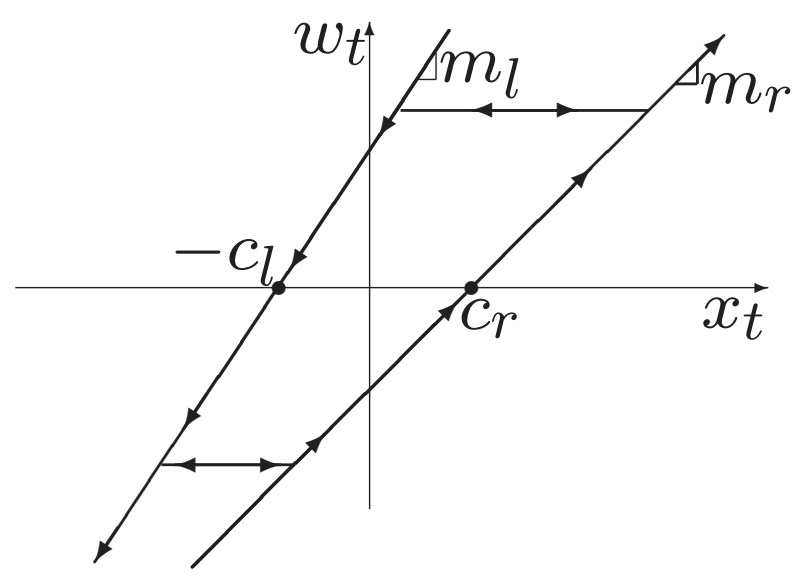

Proposition 1: If $y_{t} \in \mathcal{Y}^{r} \Rightarrow x_{t}=\frac{w_{t}}{m_{r}}+c_{r}$

Proposition 2: If $y_{t} \in \mathcal{Y}^{l} \Rightarrow x_{t}=\frac{w_{t}}{m_{l}}-c_{l}$

Proposition 3: If $y_{t} \in \mathcal{Y}^{r} \cup \mathcal{Y}^{l} \Rightarrow x_{t}=\left(\frac{w_{t}}{m_{r}}+c_{r}\right) \chi_{\mathcal{Y}^{r}}\left(y_{t}\right)+\left(\frac{w_{t}}{m_{l}}-c_{l}\right) \chi_{\mathcal{Y}^{l}}\left(y_{t}\right)$

$$
\Rightarrow m_{r} m_{l} x_{k}=m_{l}\left(y_{k}-\eta_{k}+m_{r} c_{r}\right) \chi_{\mathcal{Y}^{r}}\left(y_{k}\right)+m_{r}\left(y_{k}-\eta_{k}-m_{l} c_{l}\right) \chi_{\mathcal{Y}^{l}}\left(y_{k}\right)
$$




\section{Feasible parameter set (FPS)}

The FPS $\mathcal{D}_{\gamma \theta}$ is the projection on the parameter space of the set $\mathcal{D}$ of all system parameters $\gamma-\theta$, noise samples and $\eta_{t}$ and inner signals $x_{t}$ consistent with the measurement data sequence, the assumed model structure and the error bounds, given by:

$$
\begin{aligned}
\mathcal{D}= & \left\{(\gamma, \theta, x, \eta): x_{k}=-\sum_{i=1}^{n a} a_{i} x_{k-i}+\sum_{j=1}^{n b} b_{j} u_{k-j} ;\right. \\
& m_{r} m_{l} x_{k}=m_{l}\left(y_{k}-\eta_{k}+m_{r} c_{r}\right) \chi \mathcal{Y}^{r}\left(y_{k}\right)+m_{r}\left(y_{k}-\eta_{k}-m_{l} c_{l}\right) \chi_{\mathcal{Y}^{l}}\left(y_{k}\right) ; \\
& \left.\left|\eta_{k}\right| \leq \Delta \eta_{k}, \quad k: y_{k} \in \mathcal{Y}^{r} \cup \mathcal{Y}^{l}\right\}
\end{aligned}
$$




\section{Computation of parameter bounds}

- Exact parameter bounds:

$$
\begin{aligned}
\underline{\gamma}_{k} & =\min _{(\gamma, \theta, x, \eta) \in \mathcal{D}} \gamma_{k}, \quad \bar{\gamma}_{k}=\max _{(\gamma, \theta, x, \eta) \in \mathcal{D}} \gamma_{k} \\
\underline{\theta}_{j} & =\min _{(\gamma, \theta, x, \eta) \in \mathcal{D}} \theta_{j}, \quad \bar{\theta}_{j}=\max _{(\gamma, \theta, x, \eta) \in \mathcal{D}} \theta_{j}
\end{aligned}
$$

- Parameter Uncertainty Intervals:

$$
P U I_{\gamma_{k}}=\left[\underline{\gamma}_{k} ; \bar{\gamma}_{k}\right] \quad P U I_{\theta_{j}}=\left[\underline{\theta}_{j} ; \bar{\theta}_{j}\right]
$$

Remark 1: The system parameters $\gamma-\theta$, the inner signals $x_{t}$ and the noise samples $\eta_{t}$ are decision variables in the above optimization problem $\Rightarrow$ The number of optimization variables increases with the number of measurements

Remark 2: $\mathcal{D}$ is a nonconvex set described by polynomial constraints $\Rightarrow$ exact bound computation requires to solve a set of nonconvex optimization problems 


\section{Computation of parameter bounds}

- Standard nonlinear optimization tools can not be exploited to compute bounds on $\gamma_{k}$ (resp. $\theta_{j}$ ) since they can trap in local minima

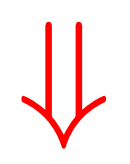

The true value is not guaranteed to lie within the computed bounds

- Relax original identification problems to convex optimization problems

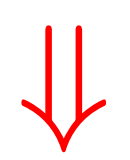

Guaranteed (relaxed) bounds on each parameter $\gamma_{k}$ (resp. $\theta_{j}$ ) can be evaluated 


\section{Computation of relaxed PUI: LMI relaxation}

- General ldea

Exploit LMI relaxation for semialgebraic optimization problems

SOS decomposition (G. Chesi et al. (1999), P. Parrillo (2003) )

Theory of moments (J. B. Lasserre (2001))

- Computational complexity

Due to the large number of optimization variables and constraints involved in the identification problems, such LMI relaxation techniques leads, in general, to untractable SDP problems

The peculiar structured sparsity of the formulated identification problems can be used to reduce the computational complexity of such LMI-relaxation techniques in computing parameter bounds 


\section{Computation of relaxed bounds: exploiting sparsity}

$$
\begin{aligned}
\mathcal{D}= & \left\{(\gamma, \theta, x, \eta): x_{k}=-\sum_{i=1}^{n a} a_{i} x_{k-i}+\sum_{j=1}^{n b} b_{j} u_{k-j} ;\right. \\
& m_{r} m_{l} x_{k}=m_{l}\left(y_{k}-\eta_{k}+m_{r} c_{r}\right) \chi \mathcal{Y}^{r}\left(y_{k}\right)+m_{r}\left(y_{k}-\eta_{k}-m_{l} c_{l}\right) \chi_{\mathcal{Y}^{l}}\left(y_{k}\right) ; \\
& \left.\left|\eta_{k}\right| \leq \Delta \eta_{k}, \quad k: y_{k} \in \mathcal{Y}^{r} \cup \mathcal{Y}^{l}\right\}
\end{aligned}
$$

- $x_{k}=-\sum_{i=1}^{n a} a_{i} x_{k-i}+\sum_{j=1}^{n b} b_{j} u_{k-j}$ only depends on the linear system parameters $a_{i}$ and $b_{j}$ and on the inner signal samples $x_{k}, \ldots, x_{k-n a}$

- $m_{r} m_{l} x_{k}=m_{l}\left(y_{k}-\eta_{k}+m_{r} c_{r}\right) \chi_{\mathcal{Y}^{r}}\left(y_{k}\right)+m_{r}\left(y_{k}-\eta_{k}-m_{l} c_{l}\right) \chi_{\mathcal{Y}^{l}}\left(y_{k}\right)$ only depends on the backlash parameters $m_{l}, c_{l}, m_{r}, c_{r}$ and on noise sample $\eta_{k}$

- $\left|\eta_{k}\right| \leq \Delta \eta_{k}$ only depends on the noise sample $\eta_{k}$ 


\section{Main properties of the proposed bounding algorithm}

Property 1 (Guaranteed relaxed uncertainty intervals)

The true parameter $\gamma_{k}$ is guaranteed to lie within the computed interval $P U I_{\gamma_{k}}^{\delta}$

\section{Property 2 (Monotone convergence to tight uncertainty intervals)}

The relaxed interval $P U I_{\gamma_{k}}^{\delta}$ monotonically converges to the tight interval $P U I_{\gamma_{k}}$ as the deep of the relaxation $\delta$ increases

\section{Property 3 (Computational complexity)}

Identification problems with more than 3000 measurements can be dealt with

Remark: The same properties also hold for $P U I_{\theta_{j}}^{\delta}=\left[\underline{\theta}_{j}^{\delta} ; \bar{\theta}_{j}^{\delta}\right]$ 


\section{Example}

\section{Simulated system}

- $\mathcal{B}: \gamma^{T}=\left[m_{r}, c_{r}, m_{l}, c_{l}\right]=[0.247,0.035,0.251,0.069]$

- $\mathcal{L}$ : second order model with parameters $\left[a_{1}, a_{2}, b_{1}, b_{2}\right]=[1.7,0.9,2.1,1.5]$.

The input is a random sequence uniformly distributed between $[-1,+1]$.

\section{Measurements errors}

- $w_{t}$ is corrupted by random additive noise, uniformly distributed between $\left[-\Delta \eta_{t},+\Delta \eta_{t}\right]$

- error bounds $\Delta \eta_{t}$ are such that $S N R_{w}=15 \mathrm{db}$.

Length of measurement data sequence: $N=2000$ 


\section{Example}

\begin{tabular}{ccccc}
\hline Parameter & $\underline{\gamma}_{i}^{\delta}$ & $\begin{array}{c}\text { True } \\
\text { Value }\end{array}$ & $\bar{\gamma}_{i}^{\delta}$ & $\Delta \gamma_{i}$ \\
\hline$m_{r}$ & 0.238 & 0.247 & 0.256 & 0.009 \\
$c_{r}$ & 0.033 & 0.035 & 0.036 & 0.002 \\
$m_{l}$ & 0.239 & 0.251 & 0.261 & 0.010 \\
$c_{l}$ & 0.065 & 0.069 & 0.073 & 0.004 \\
\hline
\end{tabular}

\begin{tabular}{ccccc}
\hline Parameter & $\underline{\theta}_{j}^{\delta}$ & $\begin{array}{c}\text { True } \\
\text { Value }\end{array}$ & $\bar{\theta}_{j}^{\delta}$ & $\Delta \theta_{j}$ \\
\hline$a_{1}$ & 1.692 & 1.700 & 1.711 & 0.009 \\
$a_{2}$ & 0.888 & 0.900 & 0.912 & 0.012 \\
$b_{1}$ & 2.035 & 2.100 & 2.161 & 0.063 \\
$b_{2}$ & 1.438 & 1.500 & 1.562 & 0.062 \\
\hline
\end{tabular}




\section{Conclusion}

- We presented a one-shot procedure for bounded-error identification of linear systems with backlash nonlinearity

- The proposed approach does not require any constraint on the input signals

- Computation of parameter bounds is formulated in terms of (nonconvex) polynomial optimization

- Guaranteed bounds are computed approximating the global optima by means of suitable (convex) LMI relaxation techniques

- Sparsity structure of the formulated problem is used to significantly reduce the computational complexity of the SDP relaxed problems.

- Convergence to tight bounds is guaranteed 